\title{
Double Laplace Decomposition Method and Finite Difference Method of Time-fractional Schrödinger Pseudoparabolic Partial Differential Equation with Caputo Derivative
}

\author{
Mahmut Modanli $\mathbb{D}^{1}$ and Bushra Bajjah $\mathbb{D}^{2}$ \\ ${ }^{1}$ Harran University, Faculty of Arts and Sciences, Department of Mathematics, 63300 Sanliurfa, Turkey \\ ${ }^{2}$ Thamar University, Faculty of Applied Sciences, Department of Mathematics, Dhamar, Yemen \\ Correspondence should be addressed to Bushra Bajjah; bushra.bajjah@tu.edu.ye
}

Received 28 July 2021; Accepted 1 October 2021; Published 23 October 2021

Academic Editor: Mehmet Emir Koksal

Copyright (c) 2021 Mahmut Modanli and Bushra Bajjah. This is an open access article distributed under the Creative Commons Attribution License, which permits unrestricted use, distribution, and reproduction in any medium, provided the original work is properly cited.

In this paper, an initial-boundary value problem for a one-dimensional linear time-dependent fractional Schrödinger pseudoparabolic partial differential equation with Caputo derivative of order $\alpha \in(0,1]$ is being considered. Two strong numerical methods are employed to acquire the solutions to the problem. The first method used is the double Laplace decomposition method where closed-form solutions are obtained for any $\alpha \in(0,1]$. As the second method, the implicit finite difference scheme is applied to obtain the approximate solutions. To clarify the performance of these two methods, numerical results are presented. The stability of the problem is also investigated.

\section{Introduction}

The initial-boundary value problem for one-dimensional pseudoparabolic partial differential equation (PPPDE) is considered as follows:

$$
u_{t}(x, t)=\lambda u_{x x t}(x, t)+k u_{x x}(x, t),
$$

with the same initial-boundary conditions mentioned for one-dimensional parabolic partial differential equation [1]:

$$
u_{t}(x, t)=k u_{x x}(x, t) .
$$

Equation (1) has been established considering the hydrostatic overflow pressure within a portion of mud during unification, where the parameter $\lambda$ is a composite soil property with the dimensions of viscosity [2]. With regard to the assumption, if the resistance to compression is elastic (proportional to the rate of compression), then equation (1) will result with $\lambda>0$. On the contrary, equation (2) is led by the classic Terzaghi hypothesis, which presumes that any increase in hydrostatic overflow pressure is proportional to an increase in the ratio of pore volume to solids' volume in mud [3].

PPPDEs appear in several fields of mechanics and physics. They are used to investigate homogeneous fluid flow in fractured rocks, quasi-stationary paths in semiconductors, thermodynamic and transportation phenomena, and different physical systems (see, e.g., [4-6]).

In the next step, the initial-boundary value problem for one-dimensional linear time-dependent Schrödinger pseudoparabolic partial differential equation (SPPPDE) in the domain $[0, L] \times[0, T]$ is considered as follows: 


$$
\begin{cases}i u_{t}(x, t)=\lambda u_{x x t}(x, t)+u_{x x}(x, t)+f(x, t), & (x, t) \in(0, L) \times(0, T] \\ u(x, 0)=\phi(x), & x \in[0, L], \\ u(0, t)=u(L, t)=0, & t \in[0, T]\end{cases}
$$

where $i=\sqrt{-1}$ is an imaginary number, $\lambda>0$, and $f$ and $\phi$ are known sufficiently smooth functions that may be complex-valued.

Since Schrödinger found his famous equation in 1926, researchers have examined the counterintuitive laws of nature for microscopic systems [7]. The time-dependent Schrödinger partial differential equation (SPDE) is originated from the vector wave equation for the electric field which rules the propagation of electromagnetic waves in an inhomogeneous medium [8]. It arises in many areas such as in quantum physics, chemistry, electromagnetic wave diffusion, underwater acoustics, and design of certain optoelectronic devices $[9,10]$.

Fractional analysis, which contains derivatives and integrals of the arbitrary order, is the generalized form of classical analysis. Recently, its applications in modeling real world phenomena have developed greatly in different fields of science and engineering, inclosing biology, physics, chemistry, medicine, finance, control theory, nanotechnology, viscoelasticity, anomalous transport, etc. The main reason for this is that the fractional modeling can be more accurate and effective, than the classical versions.

In recent years, great effort has been made to obtain analytic and approximate solutions to fractional partial differential equations (FPDEs), and numerous effective methods have been proposed, for instance, the double Laplace transform method [11], Laplace Adomian decomposition method [12], fractional iteration algorithm method [13], fractional variational iteration method [14], finite difference method [15], finite element method [16], fractional subequation method [17], modified $\left(G^{\prime} / G\right)$-expansion method [18], $\exp (\psi(\zeta))$-expansion method [19], and $q$ homotopy analysis method [20].

In this work, the initial-boundary value problem for onedimensional linear time-dependent fractional Schrödinger pseudoparabolic partial differential equation (FSPPPDE) with Caputo derivative in the domain $[0, L] \times[0, T]$ is presented as follows:

$$
\begin{aligned}
& \left\{i_{0}^{c} D_{t}^{\alpha} u(x, t)=\lambda u_{x x t}(x, t)+u_{x x}(x, t)+f(x, t), 0<\alpha \leq 1, \lambda>0,(x, t) \in(0, L) \times[0, T], u(x, 0)=\phi(x), x \in[0, L], u(0, t)\right. \\
& \quad=u(L, t)=0, t \in[0, T]
\end{aligned}
$$

where $i=\sqrt{-1}$ is an imaginary number, $f$ and $\phi$ are given sufficiently smooth and may possibly be complex-valued functions, and the Caputo derivative of order $\alpha$ is given as follows:

$$
{ }_{0}^{c} D_{t}^{\alpha} u(x, t):= \begin{cases}\frac{1}{\Gamma(m-\alpha)} \int_{0}^{t}(t-\tau)^{(m-\alpha-1)} \frac{\partial^{\alpha} u(x, \tau)}{\partial \tau^{\alpha}} \mathrm{d} \tau, & m-1<\alpha \leq m, \\ \frac{\partial^{m} u(x, t)}{\partial t^{m}}, & \alpha=m,\end{cases}
$$

where $m=[\alpha], u \in C^{m}$, and $\Gamma$ denotes Gamma function.

The double Laplace decomposition method will be constructed to solve problem (4) semianalytically. It considers one of the best integral transform utilized by many researchers. Kaabar et al. [21] defined a novel generalized double Laplace transform associated with the Adomian decomposition method and applied it to solve a reformulated nonlinear SPDE with spatiotemporal dispersion. A modified double Laplace transform decomposition method has been employed to solve the nonlinear coupled Hirota equations by Khan et al. [22]. Karatas Akgül et al. [23] utilized Laplace transform to solve the economic models based on the market balance in the sense of constant proportional Caputo derivative. Jarad and Abdeljawad [24] generalized the Laplace transform to make it viable to generalized fractional integrals and derivatives.

This work is arranged as follows. In Section 2, some necessary definitions and theorems on the double Laplace transform with an application are provided. In Section 3, the implicit finite difference scheme (FDS) for problem (4) is evoked then the stability is proved. In Section 4, we show an application with figures to illustrate the sufficiency as well as the accuracy of the approximate results. In the end, Section 5 is devoted to our conclusions. 


\section{Double Laplace Decomposition Method}

In this section, we give some basic definitions and theorems of the double Laplace transform.
Definition 1. The double Laplace transform of the function $u(x, t)$ is defined by the following double improper integral:

$$
L_{x} L_{t}[u(x, t)]:=8<U(p, s):=\int_{0}^{\infty} \int_{0}^{\infty} e^{-(p x+s t)} \mathrm{d} t \mathrm{~d} x:=\lim _{m, n \longrightarrow \infty} \int_{0}^{m} \int_{0}^{m} e^{-(p x+s t)} \mathrm{d} t \mathrm{~d} x,
$$

where $p$ and $s$ are complex numbers and $\mathfrak{R e}(p)>0$ and $\mathfrak{R e}(s)>0$.

Definition 2. If a piecewise continuous function $u(x, t)$ on the intervals $(0, X)$ and $(0, T)$ is of the exponential order, that is,

$$
|u(x, t)| \leq M e^{e_{1} x+e_{2} t}, \quad e_{1}, e_{2}>0,
$$

for a positive constant $M$, then the double Laplace transform of $u(x, t)$ exists for all $p$ and $s$ provided $\mathfrak{R e}(p)>e_{1}$ and $\mathfrak{R e}(s)>e_{2}$.
Theorem 1. Let $u \in C^{m}\left(\mathbb{R}^{+} \times \mathbb{R}^{+}\right)$and $m=\max \{n, k\}$; there exist $M, e_{1}, e_{2}>0$ such that

$$
\left|\frac{\partial^{i+j} u(x, t)}{\partial x^{i} \partial t^{j}}\right| \leq M e^{e_{1} x+e_{2} t}, \quad i=0,1, \ldots, n, j=0,1, \ldots, k .
$$

Then, the following formulas hold:

$$
\begin{aligned}
L_{x} L_{t}\left[\frac{\partial^{n} u(x, t)}{\partial x^{n}}\right]= & p^{n} U(p, s)-\sum_{i=0}^{n-1} p^{n-1-i} L_{t}\left[\frac{\partial^{i} u(0, t)}{\partial x^{i}}\right], \\
L_{x} L_{t}\left[\frac{\partial^{k} u(x, t)}{\partial t^{k}}\right]= & s^{k} U(p, s)-\sum_{j=0}^{k-1} s^{k-1-j} L_{x}\left[\frac{\partial^{j} u(x, 0)}{\partial t^{j}}\right], \\
L_{x} L_{t}\left[\frac{\partial^{n+k} u(x, t)}{\partial x^{n} \partial t^{k}}\right]= & p^{n} s^{k}\left[U(p, s)-\sum_{i=0}^{n-1} p^{-1-i} L_{t}\left[\frac{\partial^{i} u(0, t)}{\partial x^{i}}\right]\right. \\
& \left.-\sum_{j=0}^{k-1} s^{-1-j} L_{x}\left[\frac{\partial^{j} u(x, 0)}{\partial t^{j}}\right]+\sum_{i=0}^{n-1} \sum_{j=0}^{k-1} p^{-1-i} s^{-1-j} \frac{\partial^{i+j} u(0,0)}{\partial x^{i} \partial t^{j}}\right] .
\end{aligned}
$$

Theorem 2. If the Laplace transforms of $u$ and transform of the partial fractional Caputo derivative with $\left(\partial^{j} u / \partial t^{j}\right), j=0,1, \ldots, k$, exist, then the double Laplace

$$
L_{x} L_{t}\left[{ }_{0}^{c} D_{t}^{\alpha} u(x, t)\right]=s^{\alpha}\left[U(p, s)-\sum_{j=0}^{k-1} s^{-j-1} L_{x}\left[\frac{\partial^{j} u(x, 0)}{\partial t^{j}}\right]\right] .
$$

2.1. Application of the Method. We consider the following one-dimensional linear time-dependent FSPPPDE in terms of Caputo derivative:

$$
i_{0}^{c} D_{t}^{\alpha} u(x, t)=\lambda u_{x x t}(x, t)+u_{x x}(x, t)+f(x, t), \quad 0<\alpha \leq 1, \lambda>0, x>0, t>0,
$$


where

$$
f(x, t)=\left[1+t^{\alpha}+\lambda \alpha t^{\alpha-1}+i \Gamma(\alpha+1)\right] \sin (x),
$$

subject to the initial condition,

$$
u(x, 0)=\sin (x),
$$

and boundary conditions,

$$
u(0, t)=u(\pi, t)=0 .
$$

Applying the double Laplace transform with respect to $x$ and $t$ to equation (11) yields

$$
L_{x} L_{t}[u(x, t)]=\left[\frac{1}{s}+\frac{1}{i s^{\alpha+1}}+\frac{\Gamma(\alpha+1)}{s^{\alpha+1}}+\frac{\Gamma(\alpha+1)}{i s^{2 \alpha+1}}+\frac{\lambda \Gamma(\alpha+1)}{i s^{2 \alpha}}\right] \frac{1}{p^{2}+1}+\frac{1}{i s^{\alpha}} L_{x} L_{t}\left[\lambda u_{t x x}(x, t)+u_{x x}(x, t)\right] .
$$

Taking the inverse double Laplace transform to both sides of equation (15), it results in

$$
u(x, t)=\left[1+\frac{1}{i \Gamma(\alpha+1)} t^{\alpha}+t^{\alpha}+\frac{\Gamma(\alpha+1)}{i \Gamma(2 \alpha+1)} t^{2 \alpha}+\frac{\lambda \Gamma(\alpha+1)}{i \Gamma(2 \alpha)} t^{2 \alpha-1}\right] \sin (x)+L_{x}^{-1} L_{t}^{-1}\left[\frac{1}{i s^{\alpha}} L_{x} L_{t}\left[\lambda u_{t x x}(x, t)+u_{x x}(x, t)\right]\right]
$$

Using the decomposition series for $u(x, t)$ into equation (16) leads to

$$
\begin{aligned}
& \sum_{n=0}^{\infty} u_{n}(x, t)=\left[1+\frac{1}{i \Gamma(\alpha+1)} t^{\alpha}+t^{\alpha}+\frac{\Gamma(\alpha+1)}{i \Gamma(2 \alpha+1)} t^{2 \alpha}+\frac{\lambda \Gamma(\alpha+1)}{i \Gamma(2 \alpha)} t^{2 \alpha-1}\right] \\
& \sin (x)+L_{x}^{-1} L_{t}^{-1}\left[\frac{1}{i s^{\alpha}} L_{x} L_{t}\left[\lambda \frac{\partial^{3}}{\partial t \partial x^{2}} \sum_{n=0}^{\infty} u_{n}(x, t)+\frac{\partial^{2}}{\partial x^{2}} \sum_{n=0}^{\infty} u_{n}(x, t)\right]\right]
\end{aligned}
$$

From equation (17), the following recursive relationship can be obtained:

$$
\begin{aligned}
& u_{0}(x, t)=\left[1-\frac{i}{\Gamma(\alpha+1)} t^{\alpha}+t^{\alpha}-i \frac{\Gamma(\alpha+1)}{\Gamma(2 \alpha+1)} t^{2 \alpha}-i \lambda \frac{\Gamma(\alpha+1)}{\Gamma(2 \alpha)} t^{2 \alpha-1}\right] \sin (x), \\
& u_{n+1}(x, t)=L_{x}^{-1} L_{t}^{-1}\left[\frac{1}{i s^{\alpha}} L_{x} L_{t}\left[\lambda \frac{\partial^{3}}{\partial t \partial x^{2}} u_{n}(x, t)+\frac{\partial^{2}}{\partial x^{2}} u_{n}(x, t)\right]\right], \quad n \geq 0 .
\end{aligned}
$$

The following formula is reached from equation (19): 


$$
\begin{aligned}
u_{1}(x, t)= & {\left[\lambda\left(\frac{1+i \Gamma(\alpha+1)}{\Gamma(2 \alpha)} t^{2 \alpha-1}+\frac{\Gamma(\alpha+1)}{\Gamma(3 \alpha)} t^{3 \alpha-1}+i \lambda \frac{\Gamma(\alpha+1)}{\Gamma(3 \alpha-1)} t^{3 \alpha-2}\right)\right.} \\
& \left.+\left(\frac{i}{\Gamma(\alpha+1)} t^{\alpha}+\frac{1+i \Gamma(\alpha+1)}{\Gamma(2 \alpha+1)} t^{2 \alpha}+i \frac{\Gamma(\alpha+1)}{\Gamma(3 \alpha+1)} t^{3 \alpha}+\lambda \frac{\Gamma(\alpha+1)}{\Gamma(3 \alpha)} t^{3 \alpha-1}\right)\right] \sin (x), \\
u_{2}(x, t)= & {\left[\lambda^{2}\left(\frac{i-\Gamma(\alpha+1)}{\Gamma(3 \alpha-1)} t^{3 \alpha-2}+i \frac{\Gamma(\alpha+1)}{\Gamma(4 \alpha-1)} t^{4 \alpha-2}-\lambda \frac{\Gamma(\alpha+1)}{\Gamma(4 \alpha-2)} t^{4 \alpha-3}\right)\right.} \\
& +\lambda\left(\left(\frac{-1}{\Gamma(2 \alpha)} t^{2 \alpha-1}+2 \frac{i-\Gamma(\alpha+1)}{\Gamma(3 \alpha)} t^{3 \alpha-1}+(i-1) \frac{\Gamma(\alpha+1)}{\Gamma(4 \alpha)} t^{4 \alpha-1}+\lambda(i-1) \frac{\Gamma(\alpha+1)}{\Gamma(4 \alpha-1)} t^{4 \alpha-2}\right)\right. \\
& +\left(\left(\frac{-1}{\Gamma(2 \alpha+1)} t^{2 \alpha}+\frac{i-\Gamma(\alpha+1)}{\Gamma(3 \alpha+1)} t^{3 \alpha}-\frac{\Gamma(\alpha+1)}{\Gamma(4 \alpha+1)} t^{4 \alpha}+i \lambda \frac{\Gamma(\alpha+1)}{\Gamma(4 \alpha)} t^{4 \alpha-1}\right)\right] \sin (x),
\end{aligned}
$$

and so on. Hence, the required analytical solution is

$$
u(x, t)=\left(1+t^{\alpha}\right) \sin (x)
$$

where the other terms vanish in the limit.

\section{Finite Difference Scheme}

The usual notations $x_{n}=n h, 0 \leq n \leq M, t_{k}=k \delta_{t}, 0 \leq k \leq N$, and $u\left(x_{n}, t_{k}\right) \equiv u_{n}^{k}$ are used, where $u_{n}^{k}$ denotes the numerical solution of the analytical solution of $u(x, t)$ at the grid points $\left(x_{n}, t_{k}\right)$.
The numerical approximation based on the Caputo derivative is defined as

$$
{ }_{0}^{c} D_{t}^{\alpha} u n^{(k)} \approx \sigma_{\alpha, \delta_{t}} \sum_{j=0}^{k} d_{j}^{(\alpha)}\left(u n^{k-j+1}-u_{n}^{k-j}\right)+O\left(\delta_{t}^{2-\alpha}\right),
$$

where $\sigma_{\alpha, \delta_{t}}=\delta_{t}^{-\alpha} / \Gamma(2-\alpha)$ and $d_{j}^{(\alpha)}=(j+1)^{1-\alpha}-j^{1-\alpha}$

Since the solution $u \in C^{m}$ and $m \in \mathbb{N}$, then the following three-layer implicit FDS can be written at any point $\left(x_{n}, t_{k}\right)$ as follows:

$$
\begin{aligned}
\left(u_{x x t}\right)_{n}^{k}=\left(u_{t x x}\right)_{n}^{k} & \\
& \approx \frac{1}{h^{2}}\left[\left(u_{t}\right)_{n+1}^{k}-2\left(u_{t}\right)_{n}^{k}+\left(u_{t}\right)_{n-1}^{k}\right]+O\left(h^{2}\right) \\
& \approx \frac{1}{\delta_{t}}\left[\frac{u_{n+1}^{k+1}-2 u_{n}^{k+1}+u_{n-1}^{k+1}}{h^{2}}-\frac{u_{n+1}^{k}-2 u_{n}^{k}+u_{n-1}^{k}}{h^{2}}\right]+O\left(\frac{\delta_{t}}{h^{2}}+h^{2}\right) .
\end{aligned}
$$

By neglecting the following discretization errors $O\left(\delta_{t}^{2-\alpha}\right), \quad O\left(\left(\delta_{t} / h^{2}\right)+h^{2}\right)$, and $O\left(h^{2}\right)$, the implicit FDS construction of problem (4) can be written as

$$
\left\{\begin{array}{l}
i \sigma_{\alpha, \delta_{t}} \sum_{j=0}^{k} d_{j}^{(\alpha)}\left(u_{n}^{k+1-j}-u_{n}^{k-j}\right) \approx \frac{\lambda}{h^{2} \delta_{t}}\left[\left(u_{n+1}^{k+1}-2 u_{n}^{k+1}+u_{n-1}^{k+1}\right)\right. \\
\left.-\left(u_{n+1}^{k}-2 u_{n}^{k}+u_{n-1}^{k}\right)\right]+\frac{1}{h^{2}}\left[u_{n+1}^{k+1}-2 u_{n}^{k+1}+u_{n-1}^{k+1}\right]+f_{n}^{k}, \\
0<n<M, 0<k \leq N, 0<\alpha \leq 1, \lambda>0, f_{n}^{k}=f\left(x_{n}, t_{k}\right), \\
u_{n}^{0}=\phi\left(x_{n}\right), 0 \leq n \leq M, \\
u_{0}^{k}=u_{M}^{k}=0,0 \leq k \leq N,
\end{array}\right.
$$


and arrange system (24) as

$$
\left\{\begin{array}{l}
i \sigma_{\alpha, \delta_{t}}\left(u_{n}^{k+1}-u_{n}^{k}\right)+i \sigma_{\alpha, \delta_{t}} \sum_{j=1}^{k} d_{j}^{(\alpha)}\left(u_{n}^{k+1-j}-u_{n}^{k-j}\right) \\
\approx \frac{\lambda}{h^{2} \delta_{t}}\left[\left(u_{n+1}^{k+1}-2 u_{n}^{k+1}+u_{n-1}^{k+1}\right)-\left(u_{n+1}^{k}-2 u_{n}^{k}+u_{n-1}^{k}\right)\right] \\
+\frac{1}{h^{2}}\left[u_{n+1}^{k+1}-2 u_{n}^{k+1}+u_{n-1}^{k+1}\right]+f_{n}^{k} \\
0<n<M, 0<k \leq N, 0<\alpha \leq 1, \lambda>0, f_{n}^{k}=f\left(x_{n}, t_{k}\right), \\
u_{n}^{0}=\phi\left(x_{n}\right), 0 \leq n \leq M \\
u_{0}^{k}=u_{M}^{k}=0,0 \leq k \leq N .
\end{array}\right.
$$

System (25) can be rewritten as follows:

$$
\left\{\begin{array}{l}
\frac{\lambda}{h^{2} \delta_{t}} u_{n+1}^{k}-\left(\frac{1}{h^{2}}+\frac{\lambda}{h^{2} \delta_{t}}\right) u_{n+1}^{k+1}-\left(\frac{2 \lambda}{h^{2} \delta_{t}}+i \sigma_{\alpha, \delta_{t}}\right) u_{n}^{k} \\
+\left(\frac{2}{h^{2}}+\frac{2 \lambda}{h^{2} \delta_{t}}+i \sigma_{\alpha, \delta_{t}}\right) u_{n}^{k+1}+\frac{\lambda}{h^{2} \delta_{t}} u_{n-1}^{k}-\left(\frac{1}{h^{2}}+\frac{\lambda}{h^{2} \delta_{t}}\right) u_{n-1}^{k+1} \\
+i \sigma_{\alpha, \delta_{t}} \sum_{j=1}^{k} d_{j}^{(\alpha)}\left(u_{n}^{k+1-j}-u_{n}^{k-j}\right) \approx f_{n}^{k} \\
0<n<M, 0<k \leq N, 0<\alpha \leq 1, \lambda>0, f_{n}^{k}=f\left(x_{n}, t_{k}\right), \\
u_{n}^{0}=\phi\left(x_{n}\right), 0 \leq n \leq M \\
u_{0}^{k}=u_{M}^{k}=0,0 \leq k \leq N .
\end{array}\right.
$$

Writing system (26) in a matrix form is demonstrated as

$$
Q u_{n+1}+G u_{n}+Q u_{n-1}=\eta_{n}
$$


where $u_{n}=\left[u_{n}^{0}, u_{n}^{1}, \ldots, u_{n}^{N}\right]^{T}, \eta_{n}=\left[\eta_{n}^{0}, \eta_{n}^{1}, \ldots, \eta_{n}^{N}\right]^{T}$, and $\eta_{n}^{k}=f_{n}^{k}=f\left(x_{n}, t_{k}\right), 0<n<M, 0<k<N$. Also, $Q$ and $G$ are two matrices of the size $(N+1) \times(N+1)$ and have the following form:

$$
\begin{aligned}
& Q=\left[\begin{array}{ccccccc}
0 & 0 & 0 & \cdots & \cdots & \cdots & 0 \\
0 & a & b & 0 & \cdots & \cdots & 0 \\
0 & 0 & a & b & 0 & \cdots & 0 \\
\vdots & \vdots & & \ddots & \ddots & & \vdots \\
\vdots & \vdots & & & \ddots & \ddots & \vdots \\
0 & 0 & \cdots & \cdots & 0 & a & b \\
0 & 0 & 0 & \cdots & \cdots & \cdots & 0
\end{array}\right], \\
& G=\left[\begin{array}{ccccccc}
1 & 0 & 0 & \cdots & \cdots & \cdots & 0 \\
-\xi_{1} & c+\gamma_{1} & d & 0 & \cdots & \cdots & 0 \\
-\xi_{2} & \gamma_{2} & c+\gamma_{1} & d & \cdots & \cdots & 0 \\
\vdots & \vdots & & \ddots & \ddots & & \vdots \\
\vdots & \vdots & & & \ddots & \ddots & \vdots \\
-\xi_{k-1} & \gamma_{k-1} & \cdots & \cdots & \gamma_{2} & c+\gamma_{1} & d \\
-\xi_{k} & \gamma_{k} & \cdots & \cdots & \cdots & \gamma_{2} & c+\gamma_{1}
\end{array}\right] \text {, }
\end{aligned}
$$

where $a=\left(\lambda / h^{2} \delta_{t}\right), \quad b=-\left(\left(1 / h^{2}\right)+\left(\lambda / h^{2} \delta_{t}\right)\right), \quad c=-(2 \lambda /$ $\left.h^{2} \delta_{t}\right), \quad d=\left(2 / h^{2}\right)+\left(2 \lambda / h^{2} \delta_{t}\right)+i \sigma_{\alpha, \delta_{t}}, \quad \xi_{n}=i \sigma_{\alpha, \delta_{t}} d_{n}^{(\alpha)}$, and $\gamma_{n}=\xi_{n}-\xi_{n-1}, 1 \leq n \leq k$.

3.1. Stability and Convergence of the FDM. To mutate the two-step FDS (27) into one-step, the modified Gauss elimination method is used to achieve

$$
u_{n}=\theta_{n+1} u_{n+1}+\beta_{n+1}, \quad n=1,2, \ldots, M-1 .
$$

By using the given Dirichlet boundary conditions in equation (29), we obtain

$$
\theta_{1} u_{1}+\beta_{1}=u_{0}
$$

Thus, it gives

$$
\begin{aligned}
& \theta_{1}=0_{(N+1) \times(N+1)}, \\
& \beta_{1}=0_{(N+1) \times 1 .}
\end{aligned}
$$

To determine the matrices $\theta_{n+1}$ and $\beta_{n+1}$, we substitute equation (29) into equation (27), and we obtain

$$
\left(Q+G \theta_{n+1}+Q \theta_{n} \theta_{n+1}\right) u_{n+1}+\left(G \beta_{n+1}+Q \beta_{n}+Q \theta_{n} \beta_{n+1}\right)=\eta_{n} .
$$

Hence, equation (32) gives

$$
\begin{aligned}
& Q+G \theta_{n+1}+Q \beta_{n} \theta_{n+1}=0, \\
& G \beta_{n+1}+Q \beta_{n}+Q \alpha_{n} \beta_{n+1}=\eta_{n} .
\end{aligned}
$$

Finally, the following equalities are acquired:

$$
\begin{aligned}
& \theta_{n+1}=-\left(G+Q \theta_{n}\right)^{-1} Q, \\
& \beta_{n+1}=\left(G+Q \alpha_{n}\right)^{-1}\left(\eta_{n}-Q \beta_{n}\right),
\end{aligned}
$$

where $n=1,2, \ldots, M-1$

Stability can be acquired by applying the method of analyzing the eigenvalues of the iteration matrices of the schemes. The maximum of the absolute value of the eigenvalues of the matrix $Q$ has been represented by $\rho(Q)$, which refers to the spectral radius of the given matrix.

Theorem 3. FDS (26) is conditionally stable subject to the following space step limitation:

$$
h>\sqrt[4]{\left(\frac{\Gamma(2-\alpha)}{\left(d_{1}^{(\alpha)}-d_{0}^{(\alpha)}\right)}\right)^{2}\left(\frac{\delta_{t}+4 \lambda}{\delta_{t}^{1-2 \alpha}}\right) .}
$$

Proof. Using the analytical methods used in [25], we have to prove that $\rho\left(\theta_{n}\right)<1, n=1,2, \ldots, M$, under condition (35).

(i) Obviously, $\rho\left(\theta_{1}\right)=0<1$.

(ii)

$$
\begin{aligned}
\rho\left(\theta_{2}\right) & =\rho\left(-G^{-1} Q\right) \leq\left\|-G^{-1} Q\right\| \leq\left\|-G^{-1}\right\|\|Q\| \\
& =\frac{\|Q\|}{\min _{1 \leq k \leq N-1}\left(\left|a_{k k}\right|-\sum_{m \neq k}^{m=1} N-1\left|a_{k m}\right|\right)} \\
& =\frac{\left|\left(\lambda / h^{2}\right) \delta_{t}\right|+\left|-\left(\left(1 / h^{2}\right)+\left(\lambda / h^{2} \delta_{t}\right)\right)\right|}{\left|\left(-2 \lambda / h^{2} \delta_{t}\right)+\left(i \delta t^{-\alpha} / \Gamma(2-\alpha)\right)\left(d_{1}^{(\alpha)}-d_{0}^{(\alpha)}\right)\right|} \\
& =\frac{\left(2 \lambda / h^{2} \delta_{t}\right)+\left(1 / h^{2}\right)}{\sqrt{\left(2 \lambda / h^{2} \delta_{t}\right)^{2}+\left(\left(\delta_{t}^{-\alpha} / \Gamma(2-\alpha)\right)\left(d_{1}^{(\alpha)}-d_{0}^{(\alpha)}\right)\right)^{2}}}<1 \\
& \Leftrightarrow h>\sqrt{\left(\frac{\Gamma(2-\alpha)}{\left(d_{1}^{(\alpha)}-d_{0}^{(\alpha)}\right)}\right)^{2}\left(\frac{\delta_{t}+4 \lambda}{\delta_{t}^{1-2 \alpha}}\right) .}
\end{aligned}
$$

(iii) Assume that $\rho\left(\theta_{n}\right)<1$ subject to condition (35) and we need to find the value of $\rho\left(\theta_{n+1}\right)<1$. We notice that 


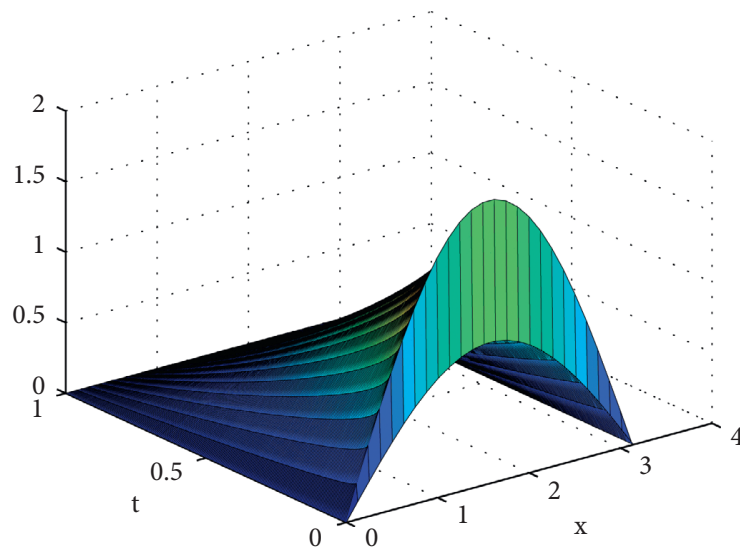

(a)

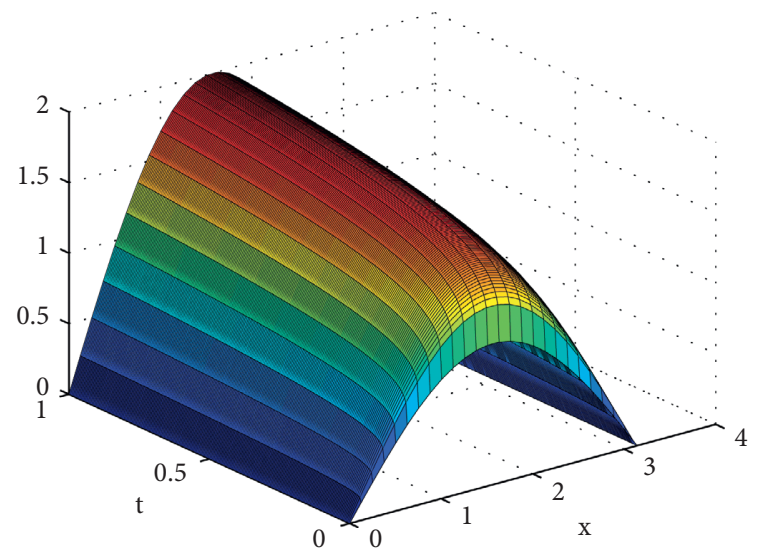

(b)

Figure 1: Profile evolution solution of problem (38) at $N=210, M=25, \alpha=0.25$, and $\lambda=2.480$. (a) Numerical simulation. (b) Analytical solution.

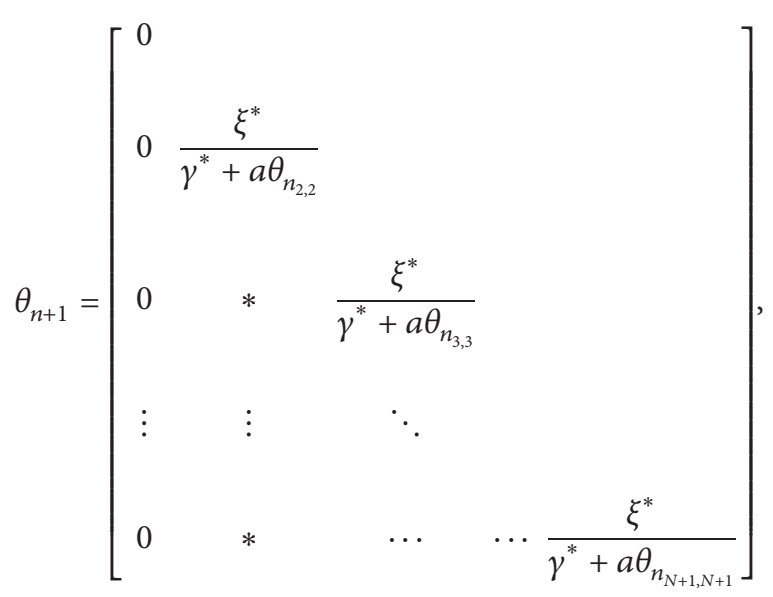

$\begin{array}{ll}\text { where } \quad \xi^{*}=\left(2 \lambda / h^{2} \delta_{t}\right)+\left(1 / h^{2}\right), & \gamma^{*}= \\ \sqrt{\left(2 \lambda / h^{2} \delta_{t}\right)^{2}+\left(\left(\delta_{t}^{-\alpha} / \Gamma(2-\alpha)\right)\left(d_{1}^{(\alpha)}-d_{0}^{(\alpha)}\right)\right)^{2}}, & \text { and }\end{array}$ $a=\left(\lambda / h^{2} \delta_{t}\right)$.

As we know that $\theta_{n_{i, i}}=\rho\left(\theta_{n}\right)$ for $2 \leq i \leq N+1$ and from the induction hypothesis, we conclude that $\rho\left(\theta_{n+1}\right)<1$ subject to condition (35). Thus, we acquire the required result by induction.

\section{Numerical Applications}

Considering the following initial-boundary value problem for one-dimensional linear time-dependent FSPPPDE in the sense of Caputo derivative,

$$
\begin{aligned}
& \left\{i_{0}^{c} D_{t}^{\alpha} u(x, t)=\lambda u_{t x x}(x, t)+u_{x x}(x, t)+f(x, t), f(x, t)\right. \\
& \quad=\left(1+t^{\alpha}+\lambda \alpha t^{\alpha-1}+i \Gamma(\alpha+1)\right) \sin (x), 0<\alpha \leq 1, \lambda>0,0<x<\pi, 0<t \leq 1, u(0, t)=u(\pi, t)=0, \quad 0 \leq t \leq 1, u(x, 0)=\sin (x), \quad 0 \leq x \leq \pi .
\end{aligned}
$$

We have shown that the analytical solution of problem

$$
u(x, t)=\left(1+t^{\alpha}\right) \sin (x)
$$
(38) is 


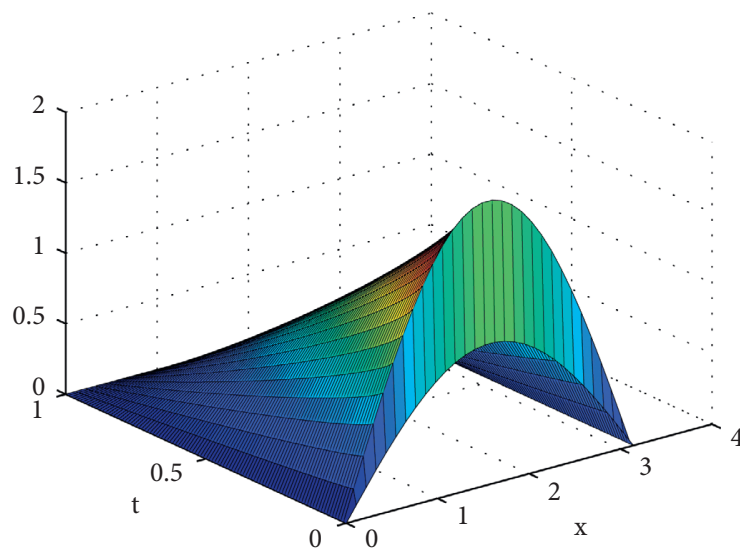

(a)

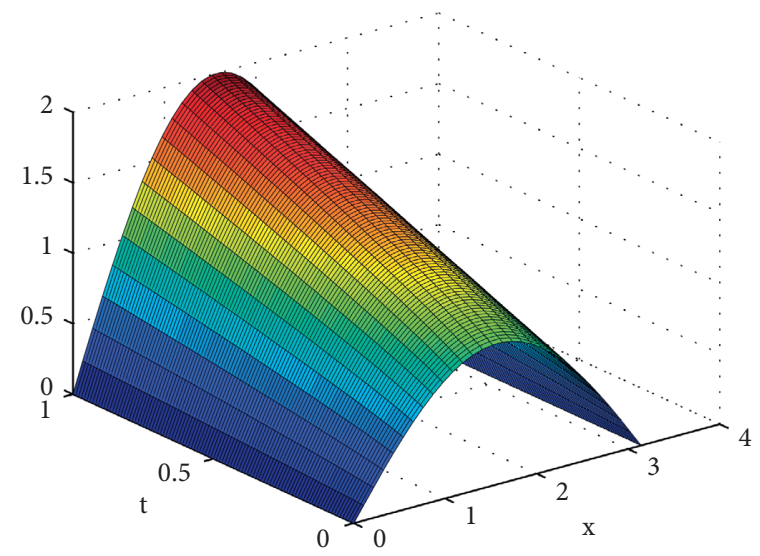

(b)

Figure 2: Profile evolution solution of problem (38) at $N=100, M=29, \alpha=0.90$, and $\lambda=2.412$. (a) Numerical simulation. (b) Analytical solution.

TABle 1: Comparison between the numerical and analytical solutions for problem (38) with $h=(\pi / M)$ and $\delta_{t}=(1 / N)$, where the chosen values of the initial data satisfy stability condition (35).

\begin{tabular}{lcccccc}
\hline$N$ & $M$ & $\alpha$ & $\lambda$ & $\begin{array}{c}\text { Numerical } \\
\text { solution }\end{array}$ & $\begin{array}{c}\text { Analytical } \\
\text { solution }\end{array}$ & $\mathcal{\varepsilon}$ \\
\hline 37 & 15 & 0.10 & 1.98 & 1.9890 & 1.9883 & 0.0008 \\
210 & 25 & 0.25 & 2.480 & 1.9979 & 1.9961 & 0.0018 \\
263 & 16 & 0.33 & 2.660 & 1.9972 & 2.0000 & 0.0028 \\
10 & 8 & 0.50 & 2.150 & 2.0003 & 2.0000 & 0.0003 \\
15 & 15 & 0.80 & 2.17 & 1.9890 & 1.9850 & 0.0040 \\
100 & 29 & 0.90 & 2.412 & 1.9981 & 1.9971 & 0.0010 \\
\hline
\end{tabular}

The maximum norm of the error of the numerical solution can be reached with

$$
\mathcal{E}=\max _{\substack{n=0,1, \ldots, M \\ k=0,1, \ldots, N}}\left|u(x, t)-u\left(x_{n}, t_{k}\right)\right|,
$$

where $u(x, t)$ and $u\left(x_{n}, t_{k}\right)$ indicate the analytical and numerical solution, respectively.

Figures 1 and 2 illustrate the comparison between the profile evolution of the analytical and numerical solutions of problem (38). The numerical solution was approximated by using implicit FDS scheme (26), whereas the analytical solution was approximated by using the double Laplace decomposition method.

We observe from Figures 1 and 2 that the numerical solution improves as the value of $\alpha$ approaches 1 (which is the case where the FPDE becomes a classical PDE). This also illustrates how conditional stability (35) of our scheme (26) is affected by the value of $\alpha$.

To quantitatively show the accuracy and efficiency of our scheme, we ran several simulations with different input parameters to compare the numerical with the analytical solutions. Table 1 shows the error $(\varepsilon)$ between the two solutions.

\section{Conclusion}

In this manuscript, the initial-boundary value problem for a one-dimensional linear time-dependent FSPPPDE in the sense of Caputo derivative of order $\alpha \in(0,1]$ is presented. Double Laplace decomposition and implicit FDS methods were investigated to obtain the closed-form and numerical solutions of problem (4), respectively. We have proved that implicit FDS (32) of problem (4) is conditionally stable. From the abovementioned table, it can be concluded that the obtained numerical solution has a good agreement with the analytic solution of various $\alpha \in(0,1]$. The results obtained in this study may be useful in many branches of quantum physics.

In the future work, we hope to extend this model to multidimensional nonlinear time-dependent coupled SPPPDEs with Caputo fractional derivatives and solve them by the finite element method.

\section{Data Availability}

No data were used to support this study.

\section{Conflicts of Interest}

The authors declare that they have no conflicts of interest.

\section{References}

[1] R. E. Showalter, "Well-posed problems for a partial differential equation of order $\$ 2 \mathrm{~m}+1 \$$," SIAM Journal on Mathematical Analysis, vol. 1, no. 2, pp. 214-231, 1970.

[2] D. W. Taylor, Research on Consolidation of Clays, Massachusetts Institute of Technology, Cambridge, MA, USA, 1942.

[3] R. E. Showalter and T. W. Ting, "Pseudoparabolic partial differential equations," SIAM Journal on Mathematical Analysis, vol. 1, no. 1, pp. 1-26, 1970.

[4] G. M. Amiraliyev, E. Cimen, I. Amirali, and M. Cakir, "Highorder finite difference technique for delay pseudo-parabolic equations," Journal of Computational and Applied Mathematics, vol. 321, pp. 1-7, 2017.

[5] A. S. Berdyshev, S. E. Aitzhanov, and G. O. Zhumagul, "Solvability of pseudoparabolic equations with non-linear boundary condition," Lobachevskii Journal of Mathematics, vol. 41, no. 9, pp. 1772-1783, 2020. 
[6] A. J. Vromans, A. A. F. van de Ven, and A. Muntean, "Parameter delimitation of the weak solvability for a pseudoparabolic system coupling chemical reactions, diffusion and momentum equations," Advances in Mathematical Sciences and Applications, vol. 28, pp. 273-311, 2019.

[7] E. Schrödinger, "An undulatory theory of the mechanics of atoms and molecules," Physical Review, vol. 28, no. 6, pp. 1049-1070, 1926.

[8] M. Dehghan, "Finite difference procedures for solving a problem arising in modeling and design of certain optoelectronic devices," Mathematics and Computers in Simulation, vol. 71, no. 1, pp. 16-30, 2006.

[9] X.-S. Zhao, Y.-R. Ge, X. Zhao, and H. Zhao, "The nature of schrodinger equation-on quantum physics Part I," 2021, https://arxiv.org/abs/2108.06198.

[10] D. Pfau, J. S. Spencer, A. G. D. G. Matthews, and W. M. C. Foulkes, "Ab initio solution of the many-electron Schrödinger equation with deep neural networks," Physical Review Research, vol. 2, no. 3, Article ID 033429, 2020.

[11] O. Ozkan and A. Kurt, "Conformable fractional double Laplace transform and its applications to fractional partial integro-differential equations," Journal of Fractional Calculus and Applications, vol. 11, no. 1, pp. 70-81, 2020.

[12] F. Haq, K. Shah, G. ur Rahman, and M. Shahzad, "Numerical solution of fractional order smoking model via laplace Adomian decomposition method," Alexandria Engineering Journal, vol. 57, no. 2, pp. 1061-1069, 2018.

[13] M. Al-Raeei and S. El-D. Moustafa, "An iteration algorithm for the time-independent fractional Schrödinger equation with Coulomb potential," Pramana, vol. 94, no. 1, pp. 1-9, 2020.

[14] Y.-J. Yang, "The local fractional variational iteration method a promising technology for fractional calculus," Thermal Science, vol. 24, no. 4, pp. 2605-2614, 2020.

[15] K. A. Mustapha, K. M. Furati, O. M. Knio, and O. P. Le Maître, "A finite difference method for space fractional differential equations with variable diffusivity coefficient," Communications on Applied Mathematics and Computation, vol. 2, no. 4, pp. 671-688, 2020.

[16] Y. Zheng and Z. Zhao, "The time discontinuous space-time finite element method for fractional diffusion-wave equation," Applied Numerical Mathematics, vol. 150, pp. 105-116, 2020.

[17] H. Yépez-Martínez and J. F. Gómez-Aguilar, "Fractional subequation method for Hirota-Satsuma-coupled KdV equation and coupled mKdV equation using the Atangana's conformable derivative," Waves in Random and Complex Media, vol. 29, no. 4, pp. 678-693, 2019.

[18] M. N. Alam and X. Li, "New soliton solutions to the nonlinear complex fractional Schrödinger equation and the conformable time-fractional Klein-Gordon equation with quadratic and cubic nonlinearity," Physica Scripta, vol. 95, no. 4, Article ID 045224, 2020.

[19] Bashar, Md Habibul, T. Tahseen, and N. H. Mahmud Shahen, "Application of the advanced $\exp (\psi(\zeta))$-Expansion method to the nonlinear conformable time-fractional partial differential equations," Turkish Journal of Mathematics and Computer Science, vol. 13, no. 1, pp. 68-80, 2021.

[20] X. Cheng, J. Hou, and L. Wang, "Lie symmetry analysis, invariant subspace method and q-homotopy analysis method for solving fractional system of single-walled carbon nanotube," Computational and Applied Mathematics, vol. 40, no. 4, pp. 1-17, 2021.

[21] M. K. A. Kaabar, F. Martínez, J. F. Gómez-Aguilar et al., "New approximate analytical solutions for the nonlinear fractional Schrödinger equation with second-order spatio-temporal dispersion via double Laplace transform method," Mathematical Methods in the Applied Sciences, vol. 44, no. 14, pp. 11138-11156, 2021.

[22] K. Khan, Z. Khan, A. Ali, and M. Irfan, "Investigation of Hirota equation: modified double laplace decomposition method," Physica Scripta, vol. 96, no. 10, Article ID 104006, 2021.

[23] E. Karatas Akgül, A. Akgül, D. Baleanu, and B. Dumitru, "Laplace transform method for economic models with constant proportional Caputo derivative," Fractal and Fractional, vol. 4, no. 3, Article ID 30, 2020.

[24] F. Jarad, T. Abdeljawad, and T. Abdeljawad, "Generalized fractional derivatives and Laplace transform," Discrete \& Continuous Dynamical Systems-S, vol. 13, no. 3, pp. 709-722, 2020.

[25] G. D. Smith, G. D. Smith, and G. D. Smith Smith, Numerical Solution of Partial Differential Equations: Finite Difference Methods, Oxford University Press, Oxford, UK, 1985. 